\title{
JÓVENES, FAMILIAS Y PROCESOS DE LITERACIDAD
}

\author{
Elisa CRAgnolino* \\ MARÍA DEL CARMEN LORENZATTI**
}

\begin{abstract}
RESUMO: Este capítulo recupera resultados de una investigación etnográfica que indagó sobre la problemática de los jóvenes pobres, su acceso a la escolaridad básica y la cultura escrita en Córdoba (Argentina). En este estudio se analizó de qué modo las políticas educativas se concretan en acciones específicas, cómo se articulan las propuestas escolares y de formación productiva y cuál es el lugar de la cultura escrita en ambos espacios. Desde los Nuevos Estudios de Literacidad (Literacy) abordaremos la presencia de las prácticas de cultura escrita en el aula y en los talleres productivos y las relaciones existentes entre la escuela y el ámbito familiar. En este sentido, se destaca la debilidad de las trayectorias escolares, el paso por diferentes instituciones, y la llegada a esta escuela como la última oportunidad de superar una historia de fracaso de varias generaciones signadas por la repitencia, la deserción, la expulsión de la escuela regular y la estigmatización.
\end{abstract}

Palabras clave: Familias. Jóvenes. Literacidad. Conocimiento.

* Doctora en Antropología y profesora de la Facultad de Filosofía y Humanidades y del Centro de Estudios Avanzados de la Universidad Nacional de Córdoba (Argentina).

E-mail: <elisag@ffyh.unc.edu.ar> <elisacagnolino@gmail.com>

** Doctora en Ciencias de La Educación y profesora de la Facultad de Filosofía y Humanidades de la Universidad Nacional de Córdoba (Argentina).

E-mail: <marial@ffyh.unc.edu.ar ><marieta.lorenzatti@gmail.com>

Cad. Cedes, Campinas, v. 33, n. 90, p. 197-214, maio-ago. 2013

Disponível em <http://www.cedes.unicamp.br> 


\section{YOUNGSTERS, FAMILIES AND LITERACY PROCESSES}

ABSTRACT: This chapter retrieves the results of an ethnographic research that investigated the problems of underprivileged youngsters, their access to basic education and the written culture in Córdoba (Argentina). In this research we seek to analyze how educational policies are materialized in specific actions, how school and production training proposals are articulated and the place written culture occupies in both spaces. Based on the New Literacy Studies we will approach the presence of written culture practices in the classroom and the workshops and the existing relationships between school and the family sphere. In this sense, the weakness of the school trajectory, frequently changing schools, and the arrival to this school as a final opportunity to overcome a history of failure of various generations marked by repetitions, leaving school before legal age, expulsion and stigma are highlighted.

Key words: Families. Youngsters. Literacy. Knowledge.

\section{Introducción}

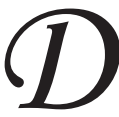

esde hace mucho tiempo los educadores de adultos están preocupados por la heterogeneidad etarea que pueblan sus aulas o espacios educativos. A partir de la V Conferencia Mundial de Educación de Adultos realizada en Hamburgo, en el año 1997, se reconoce la necesidad de nombrar a este campo como Educación de Jóvenes y Adultos porque son mayoritarios los jóvenes que buscan la posibilidad de finalizar sus estudios en otro momento de sus vidas.

En la actualidad, la presencia de los niños/jóvenes en las aulas de la escuela de adultos nos impacta a nosotros como investigadores y en las aulas desconcierta a los maestros. Docentes que, en casi su totalidad, no estuvieron preparados para trabajar en la modalidad de adultos enfrentan hoy la realidad de una escuela que recibe estudiantes cada vez jóvenes y, en algunos casos, a niños. Esta situación complejiza de manera singular la tarea docente y especialmente en relación con los procesos de literacidad.

Este capítulo recupera resultados de una investigación etnográfica ${ }^{1}$ que indagó sobre la problemática de estos jóvenes pobres, su acceso a la escolaridad básica y la apropiación de la cultura escrita en Córdoba (Argentina). Este estudio relevó las políticas e iniciativas que, desde diferentes organismos 
públicos, se desarrollan en la provincia de Córdoba y que se orientan hacia la inclusión de los jóvenes en centros educativos. En este marco se realizó un estudio en caso en una institución particular: una "Escuela Granja" que se encuentra ubicada en la zona rural de una ciudad del interior de la provincia. Este tipo de escuela forma parte de un conjunto de instituciones que intentan dar una respuesta a la problemática de los jóvenes en situación de vulnerabilidad social. Son "emergentes" que ambicionan generar un espacio distinto que articule educación y trabajo e intentan configurarse como una institución diferente del servicio brindado por la escolaridad formal de jóvenes y adultos. Nos interesó conocer, de manera particular, cuáles fueron los procesos políticos que la constituyeron como institución y de qué manera se construyó socialmente esta "propuesta alternativa" para la población juvenil. En esta línea indagamos acerca de quiénes son los jóvenes destinatarios de la propuesta y en qué medida se concreta la pretendida articulación educación básica y trabajo en esta institución.

En este capítulo presentamos reflexiones sobre la perspectiva teórica que orientó la construcción del objeto de estudio. Posteriormente, describiremos a los jóvenes estudiantes de esta institución y el lugar las prácticas de literacidad en el aula y en los talleres productivos. Por último, analizaremos las relaciones entre las prácticas de literacidad propuestas por la institución a los alumnos y las que éstos realizan en el seno familiar.

\section{De la alfabetización a la literacidad}

El concepto de alfabetización en el campo de la educación de jóvenes y adultos ha cambiado en las últimas dos décadas. Varios autores (ZAVALA, 2002; KALMAN, 2009) reconocen que el vocablo alfabetización remite a atributos negativos, como es el analfabetismo o analfabeto, que imprimen un sello estigmatizante asociado a la posición social y al nivel educativo alcanzado. En este apartado es nuestra intención presentar muy brevemente las principales referencias que orientaron el estudio. No desconocemos que son muy extensos los debates y que se trata de un campo en creciente expansión.

La preocupación por los jóvenes y el acceso a la lectura y la escritura se inscriben en una línea de trabajo que revisa las nociones tradicionales de alfabetización y se conoce como Nuevos Estudios de Literacidad (NEL). Se trata de investigaciones de tipo etnográfico que orientan su atención a 
la organización social de la lectura y escritura para dar cuenta de sus múltiples usos en los contextos locales. El concepto de literacidad destaca que la pluralidad de lo letrado es concebida como histórica y culturalmente construida y, por ende, inserta en relaciones de poder (STREET, 1993). Zavala, Niño Murcia y Ames (2004, p. 10) proponen el término literacidad como traducción de literacy porque "constituye una tecnología que está siempre inmersa en procesos sociales y discursivas, y que representa la práctica de lo letrado no sólo en programas escolares sino en cualquier contexto sociocultural". Esto es, según Street (1993), una práctica social, no solamente una habilidad técnica y neutral; esto significa que siempre se incrusta en principios epistemológicos socialmente construidos. En Brasil, desde los inicios de esta nueva perspectiva analítica se habló de letramento como traducción de literacy. Tfouni (2011) sostiene que se trata de un proceso socio-histórico, un fenómeno de ámbito cultural extenso y se refiere a las transformaciones sociales resultantes de los usos y concepciones de las personas.

Desde esta perspectiva, varios autores (STREET, 2005; BARTON; HAMILTON, 1998; HEATH, 1995) han analizado cómo la familia y los hogares se constituyen en espacios específicos donde se desarrollan prácticas letradas y cómo estas se relacionan con otros espacios comunitarios y, en particular, los escolares. Barton y Hamilton (1998) muestran en sus análisis que leer y escribir en el hogar supone muchas actividades y una multiplicidad de textos como diarios, cartas, recetas de cocina, mensajes, anuncios, textos religiosos, instrucciones sobre funcionamientos de aparatos, prescripciones médicas y folletos de medicamentos, notas varias de difrentes instituciones, entre otros. También los medios de comunicación y recursos tecnológicos, como los telefonos celulares, televisión y computadoras, suponen la incorporación a prácticas de literacidad y configuran modos particulares de relacion con la lectura y escritura. Todos esto permite plantear la complejidad de lo que sucede en el hogar en términos de literacidad, de los procesos de apropiación y de los significados de las prácticas que han sido construidas socialmente.

\section{¿De qué escuela estamos hablando?¿Quiénes son los alumnos que asisten?}

La "Escuela Granja”, objeto de nuestro estudio, fue creada en 1991 y tuvo su origen en la necesidad de dar respuesta a la problemática de los jóvenes que delinquían. Desde sus inicios la escuela contó con un centro educativo 
y con talleres (bloque, cerámica, dulces, huerta); se desarrollaban también actividades recreativas, y los chicos permanecían en la escuela en doble jornada. Durante el primer año funcionó en un galpón, en las instalaciones de una ex vidriería, en el centro de la ciudad. Luego se trasladó a un predio de 8 hectáreas, a 8 kilómetros de la ciudad. En los años siguientes, se incorporaron otros talleres (Envasado de Alimentos y el Taller de Granja) y se abrió un espacio destinado al Apoyo Pedagógico para alumnos que concurrían a instituciones de nivel secundario de la ciudad. Según nuestros análisis, la escuela transitó por tres períodos que llamamos: la creación, el apogeo y el deterioro de la institución. ${ }^{2}$

Los registros de la prensa del pasado reciente muestran algunos conflictos entre miembros que, en los diferentes tiempos de la institución, han desempeñado funciones importantes. Sucesivas notas, donde se responden acusaciones unos a otros, develan y ocultan diversos posicionamientos teóricos, metodológicos y éticos. Algunos de quienes trabajan hoy en la institución rememoran cómo trabajaron en otras épocas. En el presente pareciera añorarse el pasado prehistórico, recordando con nostalgia todo lo que pudo hacerse alguna vez.

En el momento en que realizamos el trabajo de campo (20072008), la escuela depende de la Municipalidad. El director de la Dirección de Niñez y Adolescencia cumple la función de director de la escuela y la docente pertenece a la Subdirección de Educación de Adultos (Ministerio de Educación); además trabajan psicopedagogas, personal auxiliar y un trabajador social (empleados municipales) y asisten talleristas voluntarios. También desarrollan prácticas en la institución estudiantes de psicopedagogía y trabajo social. Una de las notas distintivas en cuanto al personal, desde los comienzos de esta institución, es la precariedad y la transitoriedad. Esta situación otorga mayor fragilidad al proyecto y lo somete a los cambios de la gestión política municipal y del Ministerio de Educación de la provincia.

En el año 2008, a la escuela asistía un grupo de varones con edades que oscilan entre 11 y 16 años. No concurren mujeres a la escuela, la propia edificación se vuelve un limitante, según lo señalan dos profesionales: "[...] no hay nenas porque no hay infraestructura. Sólo tenemos un baño, ¿te imaginas? [...]”. ${ }^{3}$

Llegan hasta allí con un ómnibus que la Municipalidad destina para recogerlos en algunos barrios periféricos y trasladarlos a la escuela. La jornada se extiende desde las $8: 30 \mathrm{~h}$ hasta las $15: 30 \mathrm{~h}$. Durante la mañana se 
desarrollan las clases en las aulas y las actividades referidas a las asignaturas especiales, educación física y manualidades. Luego del almuerzo es el tiempo de los talleres productivos.

La matrícula, en ese momento, era de $35^{4}$ alumnos distribuidos en tres ciclos: en el primero, 7 alumnos (entre 11 y 15 años); en el segundo ciclo, 15 (entre 12 y 16 años) y en el tercero, 13 estudiantes (entre 14 y 16 años). Como en cualquier escuela de jóvenes y adultos, esta distribución no tiene como criterio la edad, sino los conocimientos manifestados en el examen diagnóstico que deben cumplimentar para ingresar. Como en muchos establecimientos de la modalidad, la matrícula parece ser ficticia. En nuestras visitas sólo observamos la concurrencia de 12 alumnos y su asistencia era muy irregular.

Los chicos comparten como característica común el pertenecer a familias muy pobres, con padres sin trabajo o en empleos precarios, que se encuentran bajo planes sociales, es decir reciben del Estado una asignación monetaria mínima, a cambio de ciertas prestaciones, ${ }^{5}$ pero que en cualquier caso ven comprometida seriamente su capacidad de sostenimiento cotidiano, y recurren frecuentemente al trabajo de los hijos. Algunos de los chicos están judicializados, ya sea por haber cometido delitos menores como robos de bicicletas, o por vagabundeo o por las condiciones de abandono de sus padres.

Otra característica común es que estos alumnos han sido expulsados de otros establecimientos educativos: escuelas especiales o escuelas regulares para niños. En efecto algunos chicos son derivados de escuelas especiales (establecimientos para chicos con trastornos de aprendizaje u otras "patologías"). Esta cadena de situaciones suele ubicar a los jóvenes en un circuito de códigos y de prácticas que rozan la marginalidad y que se convierten en grandes problemáticas que atraviesa la vida en la escuela granja: "las adicciones" y "la violencia".

$\mathrm{Al}$ momento de reconstruir el motivo por el cual los alumnos llegan a esta institución, encontramos cierta recurrencia. Los jóvenes indican que han sido expulsados o alejados de otras escuelas públicas; admiten que sus retiros se produjeron, principalmente, por razones de conducta tales como agresiones a sus compañeros y "faltas de respeto" a los docentes. Las instituciones educativas a la que asistían sugieren a algunos familiares que "los saquen" de esas escuelas (regulares o especiales). Luego, las relaciones familiares o de vecindad van indicando este espacio, la escuela granja, y así este establecimiento gana promoción de "boca en boca" como opción para 
los chicos con estas "difíciles" trayectorias educativas. En ocasiones llegan derivados desde la Justicia o por intervención de los servicios sociales de la Municipalidad.

La escuela se constituye así en la última opción educativa. Pero tiene además "otras ventajas": los recibe en cualquier momento del ciclo lectivo; les garantiza el transporte, les brinda almuerzo y, al menos en el imaginario familiar, les "enseña para el trabajo". Muchos de ellos no saben leer y escribir a pesar de haber finalizado el $5^{\circ}$ Grado.

\section{Las prácticas de literacidad en el aula y en los talleres ${ }^{6}$}

En este contexto institucional las prácticas de literacidad están presentes en el aula y en los talleres. Para analizar las características que asumen estas prácticas, consideramos la presencia de recursos escritos en la escuela, los contenidos de la lectura y escritura y quiénes son los actores involucrados en estas prácticas.

En relación con la disponibilidad de cultura escrita (KALMAN, 2004), se observan algunos objetos escritos en la institución, por ejemplo, la presencia de afiches en el comedor con el nombre de los alumnos; algunas pocas revistas, una de ellas referida a estrategias de enseñanza editadas para el nivel primario regular, y revistas con indicaciones para la organización de actos escolares. Mencionamos también a los espacios de escrituración aquellos recursos destinados a la escritura de alumnos y de la maestra. Ellos son: a) el pizarrón, que es el recurso más disponible en los espacios áulicos. Es la maestra quien lo emplea para transcribir las consignas de trabajo que los alumnos reproducen en sus carpetas; b) las carpetas, que son los espacios individuales que los estudiantes usan para copiar y que la docente posee para sistematizar su clase y transcribirla al pizarrón $;^{7}$ c) hojas borradores: se trata de hojas de fotocopias viejas brindadas por la maestra a los estudiantes antes de instancias de evaluación, donde responden a las consignas planteadas y después las pasan en "limpio" (las vuelven a escribir) a las carpetas; d) los manuales, libros destinados a los alumnos de cuarto y quinto grado de las escuelas regulares. No hay un libro disponible para cada joven, pero no se comparten entre los alumnos, se fomenta el trabajo de manera individual y los estudiantes respetan el turno en el uso de estos materiales; e) afiches: se usan en el aula, pegados al pizarrón. La docente copia allí fragmentos de los manuales o de su carpeta para que los alumnos transcriban en sus carpetas. 
La disponibilidad de estos recursos materiales donde los estudiantes y la maestra escriben no significa apropiación de conocimientos. Indagamos entonces de qué manera se produce el acceso (KALMAN, 2004), es decir, cuáles son las condiciones sociales que permiten a estos estudiantes apropiarse de los conocimientos, de los usos y sentidos de la literacidad.

En primer lugar, preguntamos ¿qué se escribe en esta escuela? En este sentido, nuestras observaciones muestran que se escribe sobre los contenidos escolares y éstos corresponden a distintas disciplinas. En el caso de las ciencias sociales, la escritura se relaciona con las efemérides y los actos escolares. En lengua, se abordan cuestiones gramaticales y ortográficas desligadas del sentido de la escritura. Por ejemplo, se solicita que los estudiantes señalen la cantidad de párrafos, la puntuación; otras veces, la tarea se centra en la escritura en letra cursiva. Se realizan también tareas de escritura en matemática.

Esta mirada nos conduce a otras preguntas de la investigación: ¿Quién escribe y cómo se desarrollan las prácticas de escritura en el aula? Pudimos observar que escriben los jóvenes en el aula respondiendo a las consignas de la maestra y no identificamos prácticas de escritura autónoma, donde los estudiantes desplieguen su creatividad e imaginación. En general se desarrolla un trabajo individual, donde la maestra orienta a cada estudiante. Éstos copian del pizarrón o del manual. La maestra escribe en el pizarrón y lee en voz alta. En algunas ocasiones, copian los alumnos, escribe la maestra en el pizarrón y luego lee la maestra sola en voz alta.

La copia emerge como la estrategia de enseñanza central y se copia desde el pizarrón, el afiche, las revistas, los diarios o el manual. Se copian las consignas y los ejercicios de matemática. Los chicos copian en silencio, durante un largo rato. No hay sonido en el aula más allá que el de las lapiceras deslizándose en las hojas de la carpeta de los alumnos. La copia se presenta como la única acción de escritura desarrollada por los alumnos. Es la opción privilegiada por la maestra al proponer las actividades, pero también son los alumnos quienes demandan esta práctica todos los días. Rockwell (1982, p. 306) sostiene: "la copia es práctica tan ritualizada que constituye la repuesta automática [...] cuando el maestro escribe en el pizarrón”.

Las correcciones de la maestra también se realizan en forma individual. Los niños/jóvenes lo demandan frecuentemente, necesitan la evaluación de la maestra para continuar copiando. Se observan turnos de atención, algunos copian, otros esperan la corrección del cuaderno. Son pautas de 
comportamiento escolar que se cristalizan en las escuelas de niños y en esta institución también se naturaliza.

Estos modos de literacidad muestran prácticas estáticas donde la participación de los estudiantes es nula, no se propician intercambios entre ellos para permitir expresar opiniones. Entonces nos preguntamos: ipara qué se escribe en esta escuela?

En algunos momentos, la copia de un texto, ya sea del pizarrón o del manual, cumple la función de retener al alumno en la escuela. Beinotti y Frasson (2006) sostienen que escribir y, en este caso, copiar funcionaría como un sello, como una garantía de estar en la escuela.

La copia emerge también como una estrategia de control que la maestra despliega en el aula como modo de ocupar a los alumnos en una tarea específica de la escuela: la escritura.

Estas prácticas son individuales, no se interpreta, no se discute, mucho menos se socializa en el grupo de pares. En este sentido, podemos decir que no se promueve la formación de la propia palabra de los estudiantes. La palabra propia surge de la interacción con otros. Es allí donde se aprende a reaccionar ante los textos, a usar, apreciar, criticar y significarlos mediante la convivencia con otros lectores y escritores (KALMAN, 2005). Y la escuela es el lugar, aunque no el único, donde se promueve la relación con los textos y el lenguaje escrito.

Desde lo observado, los jóvenes copian porque la docente se lo requiere; no se han registrado prácticas voluntarias de escritura, al contrario, siempre son inducidas y demandadas por la docente. Los alumnos, en mayor o en menor medida, responden a esta solicitud; algunos manifestando su desgano. La literacidad impuesta (IVANIC; MOSS, 2004) por la maestra va sesgando la participación de los alumnos en el desarrollo de sus propias prácticas escriturarias. Se puede observar una visión autónoma de la literacidad (STREET, 1993), donde los procesos se asocian más a cuestiones relacionadas con el sistema de escritura que a incentivar la escritura, recuperando los conocimientos que los estudiantes tienen de sus usos sociales. Esta posición no implica una mirada dicotómica sobre la cultura escrita en el aula y sus usos sociales, sino más bien significa reconocer que los jóvenes saben el valor y las consecuencias de la cultura escrita, pero la maestra no recupera su potencialidad para la enseñanza. En un trabajo anterior (LORENZATTI, 2011) sosteníamos que los jóvenes y adultos interactúan con otras personas y con objetos escritos todo el tiempo; leen con otros; identifican documentos 
y formularios, reconocen carteles en instituciones públicas, medicamentos, documentos a partir de distintos modos representativos; utilizan medios de comunicación y la computadora para acceder a informaciones; clasifican y organizan documentos y otros tipos de papeles (fotos, tarjetas), entre otros. Este marco posibilita pensar y trabajar la lengua como objeto de enseñanza en relación con los usos sociales de la lengua escrita.

En la escuela es innegable el alto privilegio que se le otorga al trazado o la transcripción fidedigna y meticulosa de los textos por parte de los alumnos, al momento de escribir. Expresiones como "tenés linda letra con minúscula de imprenta", "no, en los márgenes no se escribe, no queda bien" evidencian, cotidianamente, esta realidad. Coincidimos con Rockwell (1982) cuando plantea que la atención se dirige a la forma y no al contenido de aquello que se copia, se privilegia lo estético a la significación que pueda adquirir el mensaje educativo en cada alumno. El aspecto convencional de la escritura es una construcción histórica y social y no se remite al cuidado de la letra, involucra usos y prácticas alrededor de ella, en espacios específicos enmarcados en contextos sociales más amplios y responden a propósitos comunicativos de las personas. Lo que escriben los estudiantes ¿̇es leído por otras personas, ya sea en el interior de la escuela (sus pares, el director, la secretaria) o fuera de ella (sus padres, hermanos, abuelos, tíos)?. ¿Se recupera en otro lugar o momento lo que escriben los estudiantes?

En varias ocasiones observamos que los alumnos se perciben a sí mismo como incapacitados para escribir. Por ejemplo, uno de ellos, frente a un ejercicio que no puede terminar, expresa: "Dios, porque seré tan boludo para escribir". La docente reprende al alumno por no comprender la noticia, pero el niño/joven se posiciona como tonto para escribir, lo que da cuenta que su limitación está dada por la percepción de sí mismo y no por ausencia de conocimiento. Esto sesga y condiciona su lugar de escritor.

Otra pregunta que se impone es: ¿Quién lee y cómo leen los estudiantes? La maestra lee en voz alta los textos de historia, en particular, las efemérides, extraídos de los manuales de escuela primaria regular. Las prácticas de lectura de niños/jóvenes se realizan en voz baja. Se trata de una lectura silenciosa, individual. No se observaron lecturas grupales. Es la docente quien lee, resume, organiza, escribe mientras que los alumnos copian.

Resulta interesante lo planteado por Peroni (2003), quien desde un enfoque sociológico intenta conocer las prácticas de los "pocos lectores" a partir de relatos de vida de jubilados metalúrgicos y de presos. Estudia las 
distintas modalidades del encuentro con el libro, los lugares, las circunstancias y las finalidades. El autor sostiene que no hay experiencia lectora "menor", sino una "lectura estrecha" para designar a la práctica regida por un imperativo de adecuación del mundo del texto a una realidad que se opone a una lectura abierta, basada en el modelo letrado que considera el mundo del texto por sí mismo. En el caso que nos ocupa, no sólo se trata de una lectura impuesta a partir de textos que no invitan a ser leídos porque no responden a los intereses de los jóvenes, sino que la evaluación que realiza la maestra centrada en la forma de lectura va oscureciendo el lugar del contenido en la lectura. Por eso, para los estudiantes, las actividades de lectura no sólo se presentan como ajenas sino que parecen "no comprender" los significados, no "poder recordar" los contenidos y esto las convierte en densas e inabordables.

Veamos lo que sucede en otro espacio educativo de la Escuela Granja: los talleres. Además del salón de clases donde los estudiantes se acercan a los conocimientos regulares de la educación básica (matemática, lengua, ciencias sociales, entre otras), los alumnos concurren en el turno tarde a los "talleres productivos". Estos singularizaron a la Escuela Granja a lo largo de toda historia, pero en la actualidad se encuentran devaluados en cuanto a cantidad de espacios, diversidad de actividades y recursos materiales y humanos para su funcionamiento. En el momento del trabajo de campo los alumnos podían asistir sólo a tres talleres productivos. El escenario de pobreza y recorte presupuestario que afecta a la Escuela Granja se hace evidente en el hecho de que no se financia a ningún tallerista y estos espacios están a cargo de "voluntarios", personas comprometidas con la realidad de los niños y jóvenes que asisten. Regularmente, una vez a la semana, se desarrollan un taller de panadería y de huerta. Con menor frecuencia funciona un taller de Herrería, a cargo del padre de uno de los alumnos. En relación con la asistencia de los jóvenes a los talleres, ésta se encuentra sujeta a la "buena conducta" manifestada en el aula, constituyéndose de esta manera en un lugar al que sólo concurren aquellos "que se han portado bien", independientemente de los intereses manifestados por los jóvenes.

Si bien el taller se encuentra afectado por estas condiciones poco propicias, cuando indagamos cómo se construye conocimientos y se incorpora en este proceso la lectura y la escritura, observamos que la literacidad tiene un lugar diferente al que ocupa en el aula y existe también una participación distinta de los alumnos.

La lógica de funcionamiento de los talleres de carpintería y panadería implica, en general, una asistencia a clase de niños/jóvenes que no supera 
los 4 alumnos. Tal situación permite que los capacitadores puedan trabajar de una manera casi individualizada con cada uno de ellos y en un espacio donde las tareas manuales cobran un significado relevante. A lo largo de cada clase se transmiten y se ponen en juego, de manera oral, los conocimientos requeridos para el desarrollo de las tareas, como también las normas de seguridad y salubridad que demandan estas actividades. Podemos reconocer que todos los intercambios de saberes que allí se producen son resultado de conversaciones, en tono informal, entre los capacitadores y los jóvenes. Dado el carácter de las conversaciones que estructuran el trabajo en las clases de los talleres, consideramos que el tipo de textos que allí prevalece es el instruccional (MARIN, 2008).

En el taller de panadería estos textos cobran cuerpo en las recetas que se trabajan. Las mismas no se encuentran escritas en ningún recetario, afiche o cuaderno, que permanezca en la cocina, sino que el tallerista las relata a los jóvenes a medida que van realizando cada paso. En el caso de Herrería, sucede algo similar porque es él quien brinda los "paso a paso" de cada tarea, marcando el ritmo, los aciertos y anticipando los próximos movimientos de cada alumno con la soldadura.

Entendemos que la oralidad es la marca distintiva, la impronta que estructura el modo en el cual estos jóvenes se relacionan con las prácticas de literacidad en los talleres de la Escuela Granja. Priman las interacciones orales principalmente las indicaciones precisas de parte de los talleristas que van orientando un hacer concreto, inmediato, tangible. Pareciera que tiene que ver con la propia práctica y la trayectoria de los mismos y del objeto de la actividad. El interés de los niños se centra también en el hacer, en el aprender de la mecánica, los ritmos, el uso de los elementos, herramientas y "secretos" que supone el amasado del pan o de la base de pizza. Aparece entonces la imitación de las acciones estandarizadas y rutinizadas, que estarían guiadas por la necesidad de apropiarse de conocimientos pragmáticos, que además, a diferencia de lo que parece ocurrir en el aula, se les presentan como más significativos y útiles para su vida. Como veremos en el apartado siguiente, este reconocimiento hacia los talleres también estaría presente en las familias de los chicos.

Las familias, el lugar de la escuela granja y las prácticas de literacidad en el hogar

Nos interesa a continuación hacer referencia a las relaciones existentes entre el acceso a la literacidad en la escuela y el ámbito familiar. ¿¿Existen 
recursos de literacidad en las familias? ¿Éstos son reconocidos y aprovechados por la escuela?

Respecto a las familias y los estudios sociales del acceso a la cultura escrita, mencionamos que se trata de una problemática que ha sido trabajada desde la sociología y que también ha sido objeto de una vasta prepocupación en los NEL. Entre los estudios sociológicos mencionamos el de Lahire (2004), quien se ha ocupado de estudiar familias sin capital cultural para analizar las diferentes condiciones de éxito y fracaso escolar. Para explicar la mayor o menor adecuación entre los tipos de relaciones sociales y cualidades y habilidades que se fomentan en el universo escolar y el universo familiar, identifica rasgos de esta última. Uno de estos rasgos lo constituyen "las formas familiares de la cultura escrita". Reconoce, por un lado, los actos de lectura y escritura conjuntas de los padres con los hijos y, por otro lado, aquellas escrituras domésticas (lista de compras, agendas y listas de teléfonos, libros de recetas, entre otros) que contribuirían al éxito escolar, por el desarrollo que suponen de las habilidades de escritura, y también por su efecto en las disposiciones, ya que introducen una relación con el tiempo más calculadora, un cuidado del orden y la previsión y una relación reflexiva con el lenguaje.

En el trabajo de campo observamos los grupos domésticos de los alumnos de la Escuela Granja y pudimos advertir la existencia de distintas configuraciones: familias monoparentales, nucleares, ensambladas, con o sin presencia de algún otro pariente, como por ejemplo abuelos residiendo en la unidad y que se encuentran en su mayoría en el momento de expansión (cuando todos los hijos dependen del sostenimiento de los padres) o en el de fisión (cuando ya algún hijo se ha incorporado al trabajo o no reside en la unidad). Los padres tienen recorridos laborales similares y en campos restringidos. Las mujeres, que en su mayoría se incorporaron al trabajo durante la niñez, tienen empleos en servicio doméstico. Los hombres tienen trabajos precarios o empleos informales, mientras que sólo en uno de los casos el padre tiene una trayectoria más diversificada, tiene un oficio (herrero), y actualmente trabaja en una empresa. Muchos de los hijos también se incorporaron tempranamente a las actividades laborales, siempre en empleos informales, por ejemplo en cortaderos de ladrillos, a través de la venta ambulante o en actividades autogeneradas, como la limpieza de vidrios de los autos en las esquinas cuando se detienen en un semáforo o la apertura y cierre de puertas de taxis en lugares de tránsito regular, como el centro de la ciudad o la terminal de ómnibus. 
Según datos proporcionados por el trabajador social de la Escuela Granja, muchas de las familias de los alumnos que concurren a la institución cuentan con algún tipo de asistencia estatal. Esta ayuda se convierte en un recurso importante que asegura la sobrevivencia y la Escuela actúa en muchas ocasiones como mediadora para la gestión de los mismos o la obtención de ayudas adicionales (incremento en el "bolsón de mercadería" o ropa y calzado, asistencia médica), en momentos de crisis particulares.

Respecto a las trayectorias escolares se observa que la mayoría de los padres han finalizado la escolaridad primaria, aunque esta trayectoria fue interrumpida o demorada. En algunos de los progenitores aparece la intensión de un reingreso "al estudio", condicionada siempre a los requerimientos laborales y, en el caso de las mujeres, a la crianza de los hijos.

En cuanto a los hijos de las familias analizadas, se advierte que estos tuvieron recorridos educativos comunes. Se destacan la fragilidad de las trayectorias escolares, el paso por diferentes establecimientos educativos y la llegada a la escuela granja como la última oportunidad de superar una historia de fracaso de varias generaciones signadas por la repitencia, la deserción, la expulsión de la escuela regular y la estigmatización.

Para estas familias la Escuela Granja se constituye no sólo en un lugar posible, sino también un lugar deseable, porque ofrece lo que otros establecimientos educativos no han brindado a sus hijos; se trata de un lugar de contención, un lugar que los aislaría del "peligro de la calle", pero además un lugar donde aprender contenidos alternativos a los brindados por el sistema educativo regular. Se valoran así los talleres, la posibilidad de producir, trabajar con plantas y animales, desconociendo que en realidad los corrales están vacíos, no hay huerta y esos espacios prácticamente no funcionan.

Las familias parecen desconocer lo que realmente ocurre en la escuela y, a su vez, la escuela parece desconocer lo que sucede en los hogares en cuanto a recursos de cultura escrita que cotidianamente ponen en juego. Todos los días las familias interactúan con textos, algunos de sus integrantes leen y en ocasiones, aunque en menor medida, escriben de forma autónoma: hacen listas, dejan recados, escriben carteles y realizan diferentes anotaciones. Se preocupan además por supervisar, en algunos casos, las tareas escolares y repiten allí los rituales de la copia y la lectura en voz alta.

En las entrevistas realizadas y cuando indagamos sobre la disponibilidad de materiales de lectura y escritura, las familias no registran estos 
recursos. La televisión por ejemplo también está presente en los hogares, pero no se reconoce que a través de este medio se introducen mensajes escritos. Identifican en cambio como textos aquellos que están ligados directamente a lo escolar: enciclopedias, cuentos y poemas, revistas que los jóvenes obtuvieron en la Escuela Granja, cuadernos de la escuela primaria regular y del apoyo escolar. Reconocen como modalidades de acceso a la cultura escrita (KALMAN, 2004), fundamentalmente, las prácticas lectoras de tareas escolares y, en algunas oportunidades, la de la Biblia, del diario y de textos literarios que se encuentran en la casa o que los niños traen de las escuelas.

Los textos escritos que están siempre presentes en todas las casas visitadas son los cuadernos. Las prácticas de escritura están ligadas a la copia de actividades de afianzamiento de determinados contenidos escolares que presentan algunos libros o fotocopias. Podemos reconocer entonces usos de la escritura que tienen que ver con la organización doméstica, el trabajo o la recreación. Sin embargo, el carácter de "escribientes" es, en general, adjudicado a aquellos que transitan por la escolaridad y las prácticas de escritura están ligadas a la reproducción de actividades propias de las escuelas. Algunos padres, aún con grandes dificultades, intentan reproducir las estrategias tradicionales de la enseñanza escolar, fundamentalmente copiado, dictado y lectura en voz alta.

Todos estos recursos y actividades, insistimos, son ignorados por los docentes y técnicos de la escuela. Es tan fuerte el estereotipo que, por ejemplo, el trabajador social, que continuamente visita a las familias, no puede reconocer la existencia de estos recursos y prácticas en los hogares.

\section{Consideraciones finales}

En este capítulo compartimos algunos resultados de la investigación sobre las prácticas de literacidad de jóvenes/niños en la EG y la relación con las prácticas familiares. Señalamos que la escuela granja es un lugar que anuda sentidos y significados para los distintos actores que participan en ella. Para los alumnos es la escuela, demandan conocimientos, explicaciones, participación; sus familias reconocen a la escuela como un lugar de aprendizaje; a pesar de la falta de articulación de las acciones, el Estado/ las políticas sostienen cargos docentes, secretaria y técnicos, asistencia de comida y planes sociales. 
Sin embargo pareciera que todo esto no alcanza para desarrollar proyectos que consideren los intereses y las necesidades de los jóvenes. La presencia de las políticas de Estado, la enunciación de la preocupación por la "inclusión social", la voluntad de ciertos actores no logra dar continuidad y consistencia a un proyecto institucional que posibilite que los jóvenes tengan un real acceso a los conocimientos y la cultura escrita, participación educativa y ejercicio del derecho.

\section{Notas}

1. Proyecto "Escolaridad básica y cultura escrita en los jóvenes y sus familias: una trama compleja para pensar la intervención educativa”. Distinguido y subsidiado por el Programa del Centro de Cooperación Regional para la Educación de Adultos en América Latina, México (Crefal), con apoyo a la investigación en educación de jóvenes y adultos; radicado en Centro de Investigaciones Maria Saleme de Burnichon (FFYH- UNC), periodo 2007-2008.

2. En este momento es cuando se realiza el trabajo de campo de la investigación que se presenta en este artículo.

3. Charla informal con el asistente social y la psicopedagoga.

4. Según los datos obtenidos en nuestro trabajo de campo, este número es el promedio de alumnos matriculados a lo largo de los últimos 4 años.

5. Se trata del Plan Familias, cuyo objetivo es "proteger e integrar a las familias en riesgo social e incluye transferencias monetarias al titular del beneficio (el subsidio tiene una base de U\$D 50 dólares y un tope de U\$D 100), quien debe comprometerse a realizar una serie de contraprestaciones no laborables, tendientes a asegurar la asistencia escolar y los controles de salud de los menores a cargo.

6. Este apartado recupera los análisis desarrollados por la becaria Belén Uanini. Facultad de Filosofía y Humanidades (UNC).

7. En algunas oportunidades los guardan en un armario del aula, cuando salen al recreo o se retiran; este resguardo es para evitar daños en estos materiales ocasionados, principalmente, por sus compañeros. En otros momentos las carpetas quedan sobre las mesas. Sólo 2 o 3 jóvenes, por clase, se llevan sus cuadernos a la casa.

8. Tercer registro de campo (set. 2007).

9. Noveno registro de campo (oct. 2007).

\section{Referencias}

BARTON, D.; HAMILTON, M. Local literacies: reading and writing in one community. London: Routledge, 1998.

BEINOTTI G.; FRASSON M. Oralidad y escritura en las aulas de adultos. 2006. Tesis para acreditar la Licenciatura en Ciencias de la Educación, 
Escuela de Ciencias de la Educación, Facultad de Filosofía y Humanidades, Universidad Nacional de Córdoba, Córdoba.

HEATH, S. Ethnography in communities: learning the everyday life of America's subordinate youth. In: BANKS, J.Y.; MCGEE BANKS, C.A. (Ed.). Handbook of research on multicultural education. New York: MacMillan, 1995.

IVANIC, R.; MOSS, W. La incorporación de las prácticas de escritura de la comunidad en la educación. In: ZAVALA, V.; NIÑO-MURCIA, M.Y.; AMES, P. (Ed.). Escritura y sociedad: nuevas perspectivas teóricas y etnográficas. Lima: Red para el Desarrollo de las Ciencias Sociales en el Perú, 2004. p. 211-246.

KALMAN, J. Saber lo que es la letra: una experiencia de lectoescritura con mujeres de Mixquic. México, DC: Siglo XXI; SEP, 2004.

KALMAN, J. El origen social de la palabra propia. México, DF: Conaculta; Consejo Nacional para la Lectura y las Artes, 2005.

KALMAN, J. San Antonio ¡Me urge! Preguntas sin respuesta acerca de la especificidad de dominio de los géneros textuales y las prácticas letradas. In: STREET, B.; KALMAN, J. Lectura, escritura y matemáticas como prácticas sociales. México, DF: Crefal; Siglo XXI, 2009. p. 130-155.

LORENZATTI, M.C. Prácticas escolares de cultura escrita: un estudio etnográfico con adultos de nula escolaridad. In: FINNEGAM, F. (Comp.). Educación de jóvenes y adultos: políticas, instituciones y prácticas. Buenos Aires: Aique, 2011. p. 219-252.

MARIN, M. Linguística y enseñanza de la lengua. 2. ed. Buenos Aires: Aique, 2008.

PERONI, M. Historias de lectura. Trayectorias de vida y de lectura. México, DF: Fondo de Cultura Económica, 2003.

ROCKWELL, E. Los usos escolares de la lengua escrita. In: FERREIRO, E.; GOMEZ PALACIO, M. (Comp.). Nuevas perspectivas sobre los procesos de lectura y escritura. México, DF: Siglo XXI, 1982.

STREET, B. Cross-cultural approaches to literacy. Cambridge, Mass.: Cambridge University, 1993. 
STREET, B. Recent applications of New Literacy Studies in educational contexts. Research in the Teaching of English, Urbana, v. 39, n. 4, p. 417423, May 2005.

TFOUNI, L.V. Autoria e discurso pedagógico: questiones sobre o fracaso escolar. O fracasso: do sistema, o do sujeito? In: LORENZATTI, M. (Comp.). Procesos de literacidad y acceso a la educación básica de jóvenes y adultos. Unquillo: Narvaja, 2011.

ZAVALA, V. (Des)encuentros con la escritura: escuela y comunidad en los Andes peruanos. Lima: Red para el Desarrollo de las Ciencias Sociales en el Perú, 2002.

ZAVALA, V.; NIÑO-MURCIA, M.; AMES, P. (Ed.). Escritura y sociedad: nuevas perspectivas teóricas y etnográficas. Lima: Red para el Desarrollo de las Ciencias Sociales en el Perú, 2004.

Recebido em 30 de setembro de 2012.

Aprovado em 11 de abril de 2013. 